\title{
Distributed quantum dense coding
}

\author{
D. Bruß $\beta^{1}$, G.M. D'Ariano ${ }^{2}$, M. Lewenstein ${ }^{1}$, C. Macchiavello ${ }^{2}$, A. Sen(De $)^{1}$, and U. Sen ${ }^{1}$ \\ ${ }^{1}$ Institut für Theoretische Physik, Universität Hannover, D-30167 Hannover, Germany \\ ${ }^{2}$ Dipartimento di Fisica "A. Volta" and INFM-Unitá di Pavia, Via Bassi 6, 27100 Pavia, Italy
}

\begin{abstract}
We introduce the notion of distributed quantum dense coding, i.e. the generalization of quantum dense coding to more than one sender and more than one receiver. We show that global operations (as compared to local operations) of the senders do not increase the information transfer capacity, in the case of a single receiver. For the case of two receivers, using local operations and classical communication, a non-trivial upper bound for the capacity is derived. We propose a general classification scheme of quantum states according to their usefulness for dense coding. In the bipartite case (for any dimensions), bound entanglement is not useful for this task.
\end{abstract}

Entanglement is considered to be the most important resource for quantum information [1], as it allows for new quantum protocols such as superdense coding, quantum teleportation and quantum cryptography. It is therefore of great importance to classify quantum states according to their entanglement properties, in particular with respect to their usefulness for a given quantum information task. An important example of such classification concerns the distillability of quantum states, i.e. the question whether entanglement can be concentrated by local operations [2]. Recently, the question of usefulness of states for quantum teleportation [3] and quantum cryptography 跑 has been addressed.

In this Letter, we introduce the general concept of distributed dense coding (see also [5]) and present a classification of mixed states according to their dense-codeability (DC). The idea of dense coding is to use previously shared entanglement between a sender and a receiver, to send more information than that is possible without the resource of entanglement. We establish a full DCclassification for two-party systems, generalizing Refs. [6, 7, 8]. In particular, we show that bipartite bound entangled states, in any dimensions, cannot be used for dense coding. Furthermore, we consider the case of several senders and receivers, in three different scenarios: (i) the senders/receivers are distant and not allowed to communicate among themselves, (ii) they can use local operations and classical communication (LOCC), (iii) they can perform global operations. We present the classification structure for these scenarios. For the case of a single receiver, we obtain the exact DC-capacity. Surprisingly, this capacity cannot be increased by communication between the senders or their joint operations. Moreover, states which are bound entangled in the senders to receiver cut, are not useful in this scenario. For the case of more than one receiver, we obtain upper bounds for the corresponding DC-capacities.

Let us first consider the bipartite scenario. The amount of classical information that can be sent via a $d$-dimensional quantum system is at $\operatorname{most} \log _{2} d$ bits (binary digits). This is due to the Holevo bound [9]. In quantum dense coding, entanglement between the sender and receiver allows to go beyond this bound [10]. If the sender and receiver - hereafter called Alice $(\mathrm{A})$ and $\mathrm{Bob}$ (B) - share an entangled bipartite state in $d_{A} \otimes d_{B}$, Alice is sometimes able to send more than $\log _{2} d_{A}$ bits to Bob, i.e. more than the maximal information content of her subsystem without any shared entanglement. However, she certainly cannot send more than $\log _{2} d_{A}+\log _{2} d_{B}$ bits to Bob, as required by the Holevo bound.

Given a previously shared state $\rho^{A B}$ in dimension $d_{A} \otimes$ $d_{B}$, a general dense coding protocol consists of two steps.

1. Alice performs a local unitary transformation $U_{i}$ with probability $p_{i}$ on her part of $\rho^{A B}$. This means that she transforms the state $\rho^{A B}$ to the ensemble $\left\{p_{i}, \rho_{i}^{A B}\right\}$, where $\rho_{i}^{A B}=U_{i} \otimes I_{d_{B}} \rho^{A B} U_{i}^{\dagger} \otimes I_{d_{B}}$. Here $I_{d_{B}}$ is the identity operator on Bob's Hilbert space. Alice then sends her part of the ensemble state to Bob.

2. Bob extracts the maximal information about the index $i$ from the ensemble $\left\{p_{i}, \rho_{i}^{A B}\right\}$, where now the total state is at his side, by performing suitable measurements.

The maximum amount of information that Bob can gather from his measurement is bounded from above by the Holevo quantity [9]

$$
S(\bar{\rho})-\sum_{i} p_{i} S\left(\rho_{i}^{A B}\right)=\sum_{i} p_{i} S\left(\rho_{i}^{A B} \| \bar{\rho}\right) .
$$

Here $S(\varsigma)=-\operatorname{tr}\left(\varsigma \log _{2} \varsigma\right)$ denotes the von Neumann entropy, $S(\varrho \| \varsigma)=\operatorname{tr}\left(\varrho \log _{2} \varrho-\varrho \log _{2} \varsigma\right)$ is the relative entropy, and $\bar{\rho}=\sum_{i} p_{i} \rho_{i}^{A B}$. This bound can be attained asymptotically [11], so that the capacity of dense coding is defined as $\chi=\max \sum_{i} p_{i} S\left(\rho_{i}^{A B} \| \bar{\rho}\right)$, where the maximization is over all sets $\left\{U_{i}\right\}$ of unitaries performed by Alice, and all choices of probabilities $\left\{p_{i}\right\}$.

For $d_{A} \otimes d_{B}$ systems, with $d_{A}=d_{B}=d$, it was shown in Ref. [7] that the maximum is reached for a complete set of orthogonal unitary operators $\left\{W_{j}\right\}$, sampled with equal probabilities, and obeying the trace rule $\frac{1}{d^{2}} \sum_{j} W_{j}^{\dagger} \Xi W_{j}=\operatorname{tr}[\Xi] I$, for any operator $\Xi$. A typical example of such a set is provided by the group of shift-and-multiply operators $W_{(p, q)}|j\rangle=$ $\exp \left(\frac{2 \pi p j}{d}\right)|j+q(\bmod d)\rangle$, where $\{|j\rangle\}$ denotes an orthonormal basis and $p, q, j=0, \ldots, d-1$.

In a similar way one can show that the same sets of 
unitary operators with equal probabilities are also optimal for bipartite systems with $d_{A} \neq d_{B}$. Let us give a brief outline of the proof. As in Ref. [7], the optimization of the dense coding capacity proceeds in three steps.

Step 1. The average state of the ensemble $\left\{\frac{1}{d_{A}^{2}}, \rho_{j}\right\}$, that is obtained after Alice performs the unitary transformations $W_{j}$ on her subsystem, is $\bar{\rho}^{\prime}=\frac{1}{d_{A}} I_{d_{A}} \otimes \rho^{B}$, where $I_{d_{A}}$ is the identity operator on Alice's Hilbert space, and $\rho^{B}=\operatorname{tr}_{A} \rho^{A B}$. Let $\chi^{\prime}$ be the capacity for this particular choice of unitaries, so that $\chi^{\prime}=\frac{1}{d_{A}^{2}} \sum_{j} S\left(\rho_{j} \| \bar{\rho}^{\prime}\right)$.

Step 2. The capacity $\chi^{\prime}$ is equal to the relative entropy $S\left(\sigma_{A B} \| \bar{\rho}^{\prime}\right)$, for $\sigma_{A B}=U \otimes I_{d_{B}} \rho^{A B} U^{\dagger} \otimes I_{d_{B}}$, and an arbitrary unitary transformation $U$ on Alice's part.

Step 3. Consider now an arbitrary ensemble $\mathcal{E}=$ $\left\{p_{i}, \rho_{i}=U_{i} \otimes I_{d_{B}} \rho^{A B} U_{i}^{\dagger} \otimes I_{d_{B}}\right\}$ produced by unitary operators $U_{i}$ applied (with probability $p_{i}$ ) by Alice. Let $\chi_{\mathcal{E}}$ be the corresponding capacity, so that $\chi_{\mathcal{E}}=\sum_{i} p_{i} S\left(\rho_{i} \| \bar{\rho}\right)$. Since $\chi^{\prime}=S\left(\rho_{i} \| \bar{\rho}^{\prime}\right)$ for all $i$ (see Step 2), we have $\chi^{\prime}=\sum_{i} p_{i} S\left(\rho_{i} \| \bar{\rho}^{\prime}\right)$. By Donald's identity [12], $\chi^{\prime}=$ $\sum_{i} p_{i} S\left(\rho_{i} \| \bar{\rho}\right)+S\left(\bar{\rho} \| \bar{\rho}^{\prime}\right)=\chi_{\mathcal{E}}+S\left(\bar{\rho} \| \bar{\rho}^{\prime}\right)$, which is $\geq \chi_{\mathcal{E}}$, as relative entropy is a positive quantity. So this implies that the complete orthogonal set of unitaries $W_{j}$, chosen with equal probabilities, is an optimal choice for achieving the capacity for dense coding in $d_{A} \otimes d_{B}$ systems. And consequently the capacity of dense coding for a given shared state $\rho^{A B}$ is given by

$$
\chi=\log _{2} d_{A}+S\left(\rho^{B}\right)-S\left(\rho^{A B}\right) .
$$

The quantity $\chi$ could be increased when Alice and Bob were allowed to locally operate on the shared state. However, an increase of $\chi$ (e.g. via filtering) would require classical communication between them. As classical information (which is sent from the sender Alice to the receiver Bob) is the result of the dense coding protocol, we cannot allow them to perform classical communication to effect a change of the shared state.

A classical protocol (i.e. a protocol that does not require a shared quantum state) can be used by Alice to send at most $\log _{2} d_{A}$ bits of classical information. A shared quantum state is thus said to be useful for dense coding or dense-codeable (DC), if the corresponding capacity is more than $\log _{2} d_{A}$. From Eq. (2), it is clear that such states are precisely those for which $S\left(\rho^{B}\right)>S\left(\rho^{A B}\right)$, i.e. states which are more mixed locally than globally. For separable states, this inequality is never satisfied [13]. We show that even bound entangled states 14 in $d_{A} \otimes d_{B}$, i.e. states that are entangled, and yet they are not distillable, i.e. it is not possible to obtain maximally entangled states from them by LOCC, cannot be used for dense coding. For $d \otimes d$ systems, this was pointed out in Ref. 15.

Let us first state the reduction criterion [16] for detecting distillable states: If a state $\rho^{A B}$ is separable or bound entangled, then $\rho^{A} \otimes I_{d_{B}} \geq \rho^{A B}$ and $I_{d_{A}} \otimes \rho^{B} \geq \rho^{A B}$. There exist distillable states that violate this criterion.
Any state $\rho^{A B}$ for which $S\left(\rho^{B}\right)>S\left(\rho^{A B}\right)$ violates the reduction criterion [17] (see also [18]), and is hence distillable. Thus, $S\left(\rho^{B}\right)>S\left(\rho^{A B}\right)$ is not satisfied by any bound entangled state: Bipartite bound entanglement is not useful for dense coding. Note also that one cannot use a bound entangled state either to obtain a higher fidelity than classically, in a teleportation protocol [3, 19.

This concludes our studies of bipartite dense coding, where the capacity for any given composite state is described by Eq. (2). Note that any pure entangled bipartite state is useful for dense coding, whereas there exist mixed entangled states, even in dimension $2 \otimes 2$, which are not - e.g. a Werner state with singlet fraction less than $\approx .7476$. By contrast, all entangled states in $2 \otimes 2$ and $2 \otimes 3$ are useful for teleportation [3]. This shows that teleportation and dense coding are inequivalent tasks. In higher dimensions, at least the distillable states that violate the reduction criterion [16], are useful for teleportation. This is because, states that violate the reduction criterion, either already have nonclassical teleportation fidelity, or can be transformed into such a state, by single-side single-copy filtering operations [3. Moreover, DC states violate the reduction criterion [17, and hence are useful for teleportation.

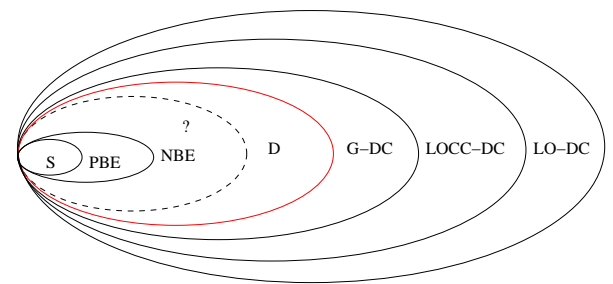

FIG. 1: Classification of multipartite quantum states, according to their usefulness for dense coding with more than one receiver. S, PBE, NBE, D stand respectively for separable, bound entangled states with positive partial transpose, bound entangled states with nonpositive partial transpose (if existing), distillable non-G-DC states (each with respect to the bipartite split between the senders and receivers); see text for other notations. For a single receiver in the multiparty case, and for bipartite systems, there are shells for S, PBE, NBE, D, G-DC only. The NBE to D boundary is not convex, provided a certain NBE state exists [20], while the convexity of G-DC to LOCC-DC boundary remains an open problem. Other boundaries are convex. In particular, the convexity of the D to G-DC boundary follows from [21].

We will now consider a scheme of dense coding for multipartite states, starting with the case of a single receiver. Suppose that there are $N-1$ Alices, say, $A_{1}, A_{2}, \ldots A_{N-1}$ and a single Bob (B). The Alices want to send (classical) information to Bob. The information of one Alice will in general be different from another Alice. To do this, they use a previously shared $N$-party state $\rho^{A_{1} \ldots A_{N-1} B}$. To start, the $j$ th Alice $A_{j}$ chooses the unitary tranformation $U_{i_{j}}^{A_{j}}$ with probability $p_{i_{j}}^{A_{j}}$, and applies it on her part of the state $\rho$. After performing the unitary trans- 
formations, the Alices send their respective parts to Bob. Then Bob makes a global measurement on the total system, to gather maximal information about Alices' ensemble. Here, Bob has no restriction in optimizing over the global measurement, and the Holevo quantity is defined by Alices' action. Note that the Holevo bound can be achieved asymptotically for product encodings of the signal states [11]. Therefore it can be reached asymptotically also in the present case of many Alices at distant locations. From the complete orthogonal set $\left\{W_{j_{l}}^{A_{l}}\right\}$ for $A_{l}$ we can construct the set of local operators $\otimes_{l} W_{j_{l}}^{A_{l}}$ which is a complete and orthogonal set for the composite system of all Alices, whence the trace rule holds for their global Hilbert space. Then, the situation is equivalent to the previous case of a single Alice. Using Steps 2, 3, discussed for $d_{A} \otimes d_{B}$ systems, it follows that the capacity of distributed dense coding with a single receiver is

$$
\begin{aligned}
\chi^{A_{1} \ldots A_{N-1} B}= & \log _{2} d_{A_{1}}+\ldots+\log _{2} d_{A_{N-1}} \\
& +S\left(\rho^{B}\right)-S\left(\rho^{A_{1} \ldots A_{N-1} B}\right) .
\end{aligned}
$$

Notice that the right hand side of Eq. (3) is equal to the capacity of dense coding when the Alices are together (see Eq. (2)). We have thus shown the surprising fact that the Alices do not need to perform global unitaries to attain the maximal capacity in a dense coding protocol. We conclude that, also in the present scenario, a state which is bound entangled in the bipartite cut $A_{1} \ldots A_{N-1}: B$, cannot be used for dense coding, since analogous considerations as before show that one cannot have $S\left(\rho^{B}\right)>S\left(\rho^{A_{1} \ldots A_{N-1} B}\right)$ for such a state.

We now consider the situation of several senders (called Alices, $A_{1}, \ldots, A_{N-1}$ ) and two receivers (called Bobs, $B_{1}$, $B_{2}$ ). If the receivers are distant and do not communicate, the corresponding DC-capacities are simply additive. This case is denoted in Fig. 1 as LO-DC. Let us therefore study the case where the Bobs are far apart, but are allowed to use LOCC between them, denoted as LOCCDC in Fig. 1. Here, some of the Alices, say $A_{1}, \ldots, A_{k}$, send their parts of the shared state $\rho^{A_{1} \ldots A_{N-1} B_{1} B_{2}}$ to $B_{1}$, while the rest of the Alices, $A_{k+1}, \ldots, A_{N-1}$, send their states to $B_{2}$. Finally, $B_{1}$ and $B_{2}$ share the ensemble $\left\{r_{i}, \zeta_{i}\right\}$, given by $r_{i}=p_{i_{1}}^{A_{1}} \ldots p_{i_{N-1}}^{A_{N-1}}, \zeta_{i}=U_{i_{1}}^{A_{1}} \otimes$ $\ldots \otimes U_{i_{N-1}}^{A_{N-1}} \otimes I_{d_{B_{1}}} \otimes I_{d_{B_{2}}} \rho^{A_{1} \ldots A_{N-1} B_{1} B_{2}} U_{i_{1}}^{A_{1} \dagger} \otimes \ldots \otimes$ $U_{i_{N-1}}^{A_{N-1} \dagger} \otimes I_{d_{B_{1}}} \otimes I_{d_{B_{2}}}$, where the unitary operator $U_{i_{j}}^{A_{j}}$ is applied by $A_{j}$ with probability $p_{i_{j}}^{A_{j}}$. Note that $B_{1}$ and $B_{2}$ are allowed to apply LOCC in the bipartite cut $A_{1} \ldots A_{k} B_{1}: A_{k+1} \ldots A_{N-1} B_{2}$.

Let us denote the classical information that can be obtained by the Bobs in this setting as $I_{a c c}^{L O C C}$. Its asymptotic version, maximized over all choices of unitaries and probabilities by the Alices, is the DC-capacity in this case, denoted as $\chi^{L O C C}$. A Holevo-like universal upper bound for $I_{a c c}^{L O C C}$, valid also for its asymptotic version, is known [22]. In the present case, it reads $\chi^{L O C C} \leq$ $\max \left(S\left(\bar{\zeta}^{(1)}\right)+S\left(\bar{\zeta}^{(2)}\right)-\max _{x=1,2} \sum_{i} p_{i} S\left(\zeta_{i}^{(x)}\right)\right)$, where $\bar{\zeta}^{(1)}=\operatorname{tr}_{A_{k+1} \ldots A_{N-1} B_{2}} \bar{\zeta}, \bar{\zeta}^{(2)}=\operatorname{tr}_{A_{1} \ldots A_{k+1} B_{1}} \bar{\zeta}$, with $\bar{\zeta}=\sum_{i} r_{i} \zeta_{i}$, and $\zeta_{i}^{(1)}=\operatorname{tr}_{A_{k+1} \ldots A_{N-1} B_{2}} \zeta_{i}, \zeta_{i}^{(2)}=$ $\operatorname{tr}_{A_{1} \ldots A_{k+1} B_{1}} \zeta_{i}$. The unspecified maximization is over all choices of unitaries and probabilities by the Alices.

To obtain a more useful bound, note that for any bipartite state $\varrho^{A B}$, local unitaries cannot change the spectrum of the global as well as the local density matrices. In particular, for arbitrary unitaries $U^{A}$ and $U^{B}$ acting on $\varrho^{A B}$ to obtain $\varrho^{A B}=U^{A} \otimes U^{B} \varrho^{A B} U^{A \dagger} \otimes U^{B \dagger}$, we have $S\left(\operatorname{tr}_{B} \varrho^{A B}\right)=S\left(\operatorname{tr}_{B} \varrho^{\prime A B}\right)$ and $S\left(\operatorname{tr}_{A} \varrho^{A B}\right)=$ $S\left(\operatorname{tr}_{A} \varrho^{\prime A B}\right)$. Using this fact, the bound on $\chi^{L O C C}$ can be simplified to obtain $\chi^{L O C C} \leq \max \left(S\left(\bar{\zeta}^{(1)}\right)+S\left(\bar{\zeta}^{(2)}\right)\right)-$ $\max _{x=1,2} S\left(\rho^{(x)}\right)$, where $\rho^{(1)}=\operatorname{tr}_{A_{k+1} \ldots A_{N-1} B_{2}} \rho, \rho^{(2)}=$ $\operatorname{tr}_{A_{1} \ldots A_{k+1} B_{1}} \rho$, and the unspecified maximization is as before. This maximization can be performed as follows. First, note that the maximizations for the two subsets of Alices are independent, as they concern disjoint subspaces of the Hilbert space. Thus, we have to find the maximum of the concave function $S\left(\bar{\zeta}^{(x)}\right)(x=1,2)$. Moreover, the $\bar{\zeta}^{(x)}$ form a convex set, for all choices of unitaries $U_{i_{j}}^{A_{j}}$ and probabilities $p_{i_{j}}^{A_{j}}$. Thus to achieve this maximum, it is sufficient to show that the first derivative of $S$ vanishes, because here a local maximum is the global one. Perturbation of the solution from the previous maximization tasks, namely $W_{j}$ with equal probabilities, shows in a straightforward way that this solution is again the optimal one. Thus, we arrive at

$$
\begin{aligned}
& \chi^{L O C C} \leq \log _{2} d_{A_{1}}+\ldots+\log _{2} d_{A_{N-1}} \\
& +S\left(\rho^{B_{1}}\right)+S\left(\rho^{B_{2}}\right)-\max _{x=1,2} S\left(\rho^{x}\right) \equiv \mathcal{B}^{L O C C},
\end{aligned}
$$

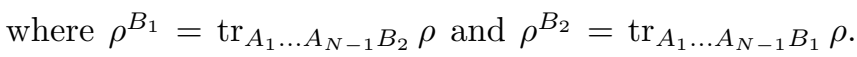
Analogous arguments as in Steps 2, 3 also give Eq. (何).

A trivial lower bound on $\chi^{L O C C}$ is given by the case where the two Bobs do not use communication; thus their two channels are independent, and the capacities add. We denote the capacity without communication as $\chi^{B_{1} B_{2}}$, and thus have $\chi^{L O C C} \geq \chi^{B_{1}}+\chi^{B_{2}}=\chi^{B_{1} B_{2}}$. A trivial upper bound on $\chi^{L O C C}$ is obtained by using the fact that the Bobs can obtain more (at least, not less) information, if they are together and are allowed to use global measurements, referred to as G-DC in Fig. 1. Let us call this bound the global DC-capacity $\chi^{\text {glob }}$ : $\chi^{L O C C} \leq \log _{2} d_{A_{1}}+\ldots+\log _{2} d_{A_{N-1}}+S\left(\rho^{B_{1} B_{2}}\right)-S(\rho)=$ $\chi^{g l o b}$. We summarize our results for the dense-codeability of a given multipartite quantum state, for two receivers, in Fig. 1. We call a state dense-codeable, if its capacity is greater than $\log _{2} d_{A_{1}}+\ldots+\log _{2} d_{A_{N-1}}$, and locally dense-codeable if $\chi^{B_{1} B_{2}}>\log _{2} d_{A_{1}}+\ldots+\log _{2} d_{A_{N-1}}$.

We now provide examples for the sets indicated in Fig. 1, and thus show that the sets are non-empty. Note also, that for these examples of DC states, one can add the 
identity to the corresponding state (up to a certain limit), and still keep the noisy state dense-codeable. Therefore the sets are not of measure zero.

An example of a state that is G-DC, but not LOCCDC (i.e. $\mathcal{B}^{L O C C} \leq \log _{2} d_{A_{1}}+\ldots+\log _{2} d_{A_{N-1}}<\chi^{\text {glob }}$ ) is $\frac{1}{2}(|0000\rangle+|0101\rangle+|1000\rangle+|1110\rangle)$ from [23], where the first two parties are senders and the last two parties are receivers, with the first (respectively, second) party sending her subsystem to the third (respectively, fourth).

The four-qubit GHZ state [24], namely $(|0000\rangle+$ $|1111\rangle) / \sqrt{2}$, is not locally DC, as the two-party reduced density matrices are separable. However, it is useful for LOCC-DC: When the two senders choose the Pauli unitaries with equal probabilities, one can show that the two receivers can completely distinguish the resulting ensemble of eight orthogonal states by LOCC. This protocol and the upper bound in Eq. (4), give $\chi^{L O C C}=3$.

A trivial example for a state that is already locally DC is the tensor product of two singlets. A four-party $W$ state 25 is not locally DC, but it is yet unknown whether it is LOCC-DC. The general problem in proving that a state is useful for LOCC dense coding is that the bound in (4) is sometimes not very tight, and can even be higher than the global bound $\chi^{g l o b}$, as is the case for the bound entangled states of Ref. [26]. The question whether there exist multipartite bound entangled states that are DC remains open. We point out here that the ordering of states that is induced by the task "dense coding", as illustrated in Fig. 1, is different from the ordering induced by other entanglement criteria, e.g. as described in [23]. Each quantum information processing objective may even lead to its own structure of quantum states.

Finally, it is formally possible to generalise these considerations to the case where there are more than two receivers. However, the main obstacle is that there is as yet no good estimation of mutual information that is accessible locally, for the case of more than two parties. For an attempt in this direction, see Ref. 27.

In summary, we have introduced the notion of densecodeability, i.e. the usefulness of a given quantum state for dense coding. We have generalized bipartite dense coding to the multipartite case, and investigated the classification of entangled states according to their dense codeability. We have presented a full classification for the bipartite case, and showed that here bound entangled states in any dimensions are not dense-codeable. In the multipartite case the capacity of dense coding depends on the possibility of interactions between the receivers. Here, we proposed a classification scheme and showed examples for the various identified classes.

We acknowledge support of the Deutsche Forschungsgemeinschaft (SFB 407, SPP 1078), the Alexander von Humboldt Foundation, and the EC Contract No. IST2002-38877 QUPRODIS. D.B and M.L. are grateful for the hospitality of the organisers of the Pavia Workshop Quantum Information Processing and Quantum Com- munications, supported by the ESF and Ministero dell'Istruzione, dell'Università e della Ricerca (Cofinanziamento 2002).

[1] D. Bouwmeester, A. Ekert, and A. Zeilinger (Eds.), The Physics of quantum information (Springer, Berlin, 2000); D. Heiss (Ed.), Fundamentals of Quantum Information, Quantum Computation, Communication, Decoherence and All That (Springer, NY, 2002).

[2] D. Bruß et al., J. Mod. Opt. 49, 1399 (2002).

[3] N. Linden and S. Popescu, Phys. Rev. A 59, 137 (1999); M. Horodecki, P. Horodecki, and R. Horodecki, Phys. Rev. A 60, 1888 (1999).

[4] D. Bruß et al., Phys. Rev. Lett. 91, 097901 (2003); M. Curty, M. Lewenstein, and N. Lütkenhaus, ibid. 92, 217903 (2004); A. Acin and N. Gisin, quant-ph/0310054.

[5] X.S. Liu, G.L. Long, D.M. Tong, and F. Li, Phys. Rev. A 65, 022304 (2002).

[6] S. Bose, M.B. Plenio, and V. Vedral, quant-ph/9810025.

[7] T. Hiroshima, quant-ph/0009048.

[8] M. Ziman and V. Bužek, Phys. Rev. A 67, 042321 (2003).

[9] J.P. Gordon, in Proc. Int. School Phys. "Enrico Fermi, Course XXXI", ed. P.A. Miles, pp. 156 (Academic Press, NY 1964); L.B. Levitin, in Proc. VI National Conf. Inf. Theory, Tashkent, pp. 111 (1969); A.S. Holevo, Probl. Pereda. Inf. 9, 31973 [Probl. Inf. Transm. 9, 110 (1973)].

[10] C.H. Bennett and S.J. Wiesner, Phys. Rev. Lett. 69, 2881 (1992).

[11] B. Schumacher and M.D. Westmoreland, Phys. Rev. A 56, 131 (1997); A.S. Holevo, IEEE Trans. Inf. Theory 44, 269 (1998).

[12] M.J. Donald, Math. Proc. Cam. Phil. Soc. 101, 363 (1987).

[13] R. Horodecki, P. Horodecki, and M. Horodecki, Phys. Lett. A 210, 377 (1996).

[14] M. Horodecki, P. Horodecki, and R. Horodecki, Phys. Rev. Lett. 80, 5239 (1998).

[15] M. Horodecki et al., QIC, 1, 70 (2001).

[16] M. Horodecki and P. Horodecki, Phys. Rev. A 59, 4206 (1999); N.J. Cerf, C. Adami, and R.M. Gingrich, ibid. 60, 898 (1999).

[17] K.G.H. Vollbrecht and M.M. Wolf, quant-ph/0202058.

[18] T. Hiroshima, Phys. Rev. Lett. 91, 057902 (2003).

[19] C.H. Bennett et al., Phys. Rev. Lett. 70, 1895 (1993).

[20] P.W. Shor, J.A. Smolin, and B.M. Terhal, Phys. Rev. Lett. 86, 2681 (2001).

[21] A. Wehrl, Rev. Mod. Phys. 50, 221 (1978).

[22] P. Badzia̧g, M. Horodecki, A. Sen(De), and U. Sen, Phys. Rev. Lett. 91, 117901 (2003).

[23] F. Verstraete, J. Dehaene, B. De Moor, and H. Verschelde, Phys. Rev. A 65, 052112 (2002).

[24] D.M. Greenberger, M.A. Horne, and A. Zeilinger, in Bell's Theorem, Quantum Theory, and Conceptions of the Universe, ed. M. Kafatos, (Kluwer, Dordrecht, 1989).

[25] W. Dür, G. Vidal, and J.I. Cirac, Phys. Rev. A 62, 062314 (2000).

[26] W. Dür, J.I. Cirac, and R. Tarrach, Phys. Rev. Lett. 83, 3562 (1999).

[27] M. Horodecki, A. Sen(De), and U. Sen, quant$\mathrm{ph} / 0310100$. 